\title{
Self-excitation of space charge waves
}

\section{Lyuksyutov, Sergei; Buchhave, Preben; Vasnetsov, Mikhail}

\section{Published in:}

Physical Review Letters

Link to article, DOI:

10.1103/PhysRevLett.79.67

Publication date:

1997

\section{Document Version}

Publisher's PDF, also known as Version of record

Link back to DTU Orbit

\section{Citation (APA):}

Lyuksyutov, S., Buchhave, P., \& Vasnetsov, M. (1997). Self-excitation of space charge waves. Physical Review Letters, 79(1), 67-70. https://doi.org/10.1103/PhysRevLett.79.67

\section{General rights}

Copyright and moral rights for the publications made accessible in the public portal are retained by the authors and/or other copyright owners and it is a condition of accessing publications that users recognise and abide by the legal requirements associated with these rights.

- Users may download and print one copy of any publication from the public portal for the purpose of private study or research.

- You may not further distribute the material or use it for any profit-making activity or commercial gain

- You may freely distribute the URL identifying the publication in the public portal

If you believe that this document breaches copyright please contact us providing details, and we will remove access to the work immediately and investigate your claim 


\title{
Self-Excitation of Space Charge Waves
}

\author{
Sergei F. Lyuksyutov,* Preben Buchhave, and Mikhail V. Vasnetsov* \\ Technical University of Denmark, Building 309, 2800 Lyngby, Denmark
}

(Received 15 January 1997)

\begin{abstract}
We report a direct observation of space charge waves in photorefractive crystals with point group 23 (sillenites) based on their penetration into an area with uniform light illumination. It is shown experimentally that the quality factor of the waves increases substantially with respect to what current theory predicts [B. Sturman et al., Appl. Phys. A 55, 235 (1992)]. This results in the appearance of strong spontaneous beams caused by space charge wave self-excitation. [S0031-9007(97)03505-9]
\end{abstract}

PACS numbers: $42.65 . \mathrm{Hw}, 42.70 . \mathrm{Nq}$

The subject of space charge waves (SCWs) embraces a wide range of physics from plasmas and semiconductors to the relatively new area of photorefractive optics $[1,2]$. In photorefractive materials SCWs manifest themselves through resonance phenomena resulting in the enhancement of space charge fields in cubic sillenite crystals with point group 23. Current ideas consider SCWs as eigen modes of the space charge field when excited with a running light grating [3] or with an ac electric field [4]. It has been suggested [5] that SCWs exist in sillenites due to a high value of mobility-lifetime product $\mu \tau$ (SturmanRinghofer model), and later it was predicted that the possibility for excitation of SCWs with some new features exists in crystals with point group $3 m$ [6]. The theory of interaction between SCWs was developed by Liberman and Zel'dovich in [7,8]. However, all experimental evidence of SCWs so far has been solely based on the detection of diffraction efficiency and gain resonances in a two-wave coupling geometry $[3,9]$.

In this Letter we describe new experiments that allow a direct observation of SCWs propagating in $\mathrm{Bi}_{12} \mathrm{SiO}_{20}$. We obtain our results for nonplanar geometry, in which the generated SCWs appear to have a maximum value of the quality factor $Q$. In this case we observe two related phenomena: Self-excitation of the SCWs and self-oscillation resulting in spontaneously generated light beams.

The SCW may be written as a running wave of the form $\exp \left(i K_{0} \xi-\gamma \xi\right)$, where $\xi=r-v_{0} t$ is the position coordinate in a frame of reference moving with the wave, $K_{0}$ is the wave number, $v_{0}$ is the velocity of the wave, and $\gamma$ is a damping factor. Suppose a crystal is illuminated with an interference field running with velocity $v$, $I_{0}[1+m \cos (K r-v t)]$, where $I_{0}$ is the total light intensity and $m$ is the modulation index. The resonance SCWs excitation occurs when $v=v_{0}$ and $K=K_{0}$. The amplitude of the forced space charge field oscillations reads [5]

$$
E_{\mathrm{sc}}=\frac{m E_{0}}{\sqrt{\left(v / v_{0}-1\right)^{2}+4 Q^{-2}\left(v / v_{0}\right)^{2}}},
$$

where $v_{0}=s I_{0} e N_{D} / \varepsilon \varepsilon_{0} E_{0} K_{0}^{2}$ is the resonance velocity, $E_{0}$ is external field, and $Q=K_{0} / \gamma=2 K_{0} \mu E_{0} / \gamma_{R} N_{A}$ is the quality factor of the SCWs. The material parameters taken from $[10,11]$ are as follows: $\mu=10^{-5} \mathrm{~m}^{2} / \mathrm{V} \mathrm{s}$ is mobility, $\gamma_{R}=1.65 \times 10^{-17} \mathrm{~m}^{3} / \mathrm{s}$ is the recombination constant, $s=0.64 \times 10^{-5} \mathrm{~m}^{2} / \mathrm{J}$ is the photoexcitation cross section, $N_{D} \approx 10^{26} \mathrm{~m}^{-3}$ is the concentration of active centers, $N_{A} \approx 10^{22} \mathrm{~m}^{-3}$ is the acceptor (negatively charged centers) concentration, $\varepsilon=56$. The optimum value of $K_{0}$ depends on the applied electric field [9] and was in our case $2.7 \times 10^{5} \mathrm{~m}^{-1}$ corresponding to a grating spacing of $23 \mu \mathrm{m}$. For the light intensity $I_{0}=10 \mathrm{~mW} / \mathrm{cm}^{2}$ and $E_{0}=6 \times 10^{5} \mathrm{~V} / \mathrm{m}$ the calculated resonance velocity amounts to $v_{0}=0.47 \mathrm{~mm} / \mathrm{s}$. Evaluation of $Q$ gives the value $Q \approx 20$. The length of SCW propagation into the shadow area (outside the running interference pattern) may be estimated as $l=\gamma^{-1}=$ $2 \mu E_{0} / \gamma_{R} N_{A}$, and thus we expect a penetration of SCWs into a shadow area for a distance of about $70 \mu \mathrm{m}$.

We performed the first observations of damped SCWs in an area without running light fringes in a traditional planar orientation, where the grating vector is along the direction of the applied electric field (as shown in Fig. 1). An external electric field $E_{0}$ was applied along the $\langle 001\rangle$

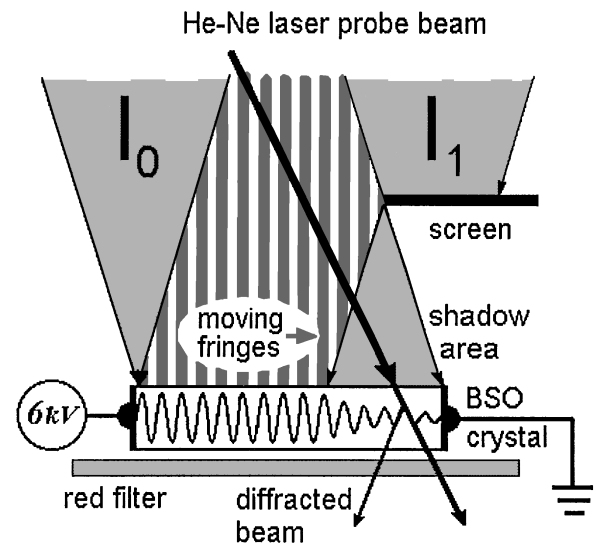

FIG. 1. Sketch of planar configuration. Two beams with intensities $I_{0}$ and $I_{1}$ excite SCW penetrating into the shadow area produced by partial blocking of the beam $I_{1}$. An expanded beam of He-Ne laser diffracting on refractive-index grating is schematically shown as a single ray. A red glass filter cuts $\mathrm{Ar}^{+}$ laser light off behind the crystal. 
crystal direction. A detailed description of the experimental setup is given in [12]. The interference fringes with contrast $m \ll 1$ (in order to have nearly the same free carrier concentration throughout the crystal) imparted on the crystal are produced by two Ar-ion laser beams at the wavelength $\lambda=0.515 \mu \mathrm{m}$. Fringes are set in motion by a small frequency detuning between the beams. Part of the weak $I_{1}$ is blocked with a screen placed before the crystal, which is $10 \times 10 \times 4 \mathrm{~mm}$ in size. Thus, no running grating exists in the part of the crystal that is receiving only uniform illumination with the intensity $I_{0}$. A near field view of the diffracted He-Ne laser beam is shown in Fig. 2(a). A sharp boundary is clearly seen between the shadow area and the area with grating. The picture changes if the fringes are set in motion. Under resonance conditions a clear dynamic pattern of "protuberances" is observed in the diffracted beam [Fig. 2(b)]. This is a qualitative proof of SCW penetration into the shadow area. The depth of penetration was about $1 \mathrm{~mm}$, indicating that $Q$ may be much higher than the calculated value. However, light scattered and diffracted from beam $I_{1}$ into the shadow area is strongly amplified in the resonance condition and may have some of the same characteristics as damped SCWs.

The next step was to perform measurements in a nonplanar orientation (Fig. 3) where the grating vector is tilted an angle $\psi$ with respect to the electric field. The deviation from the planar orientation was found to produce an increase in photorefractive gain [13]. The origin of this increase is connected with photoelastic and piezoelectric effects $[13,14]$. To find the direction of maximum $Q$ we injected a weak beam into the crystal at different angles $\psi$ and $\theta$ and measured the amplification of the beam intensity. The direction of maximum amplification was at $\psi \approx 33^{\circ}$ and $\theta \approx 1.2^{\circ}$. The measured amplification was seven times higher than for the planar configuration.

We observed some light in the shadow area related with scattering on imperfections inside the crystal of one of the beams. There was no diffracted light detected when
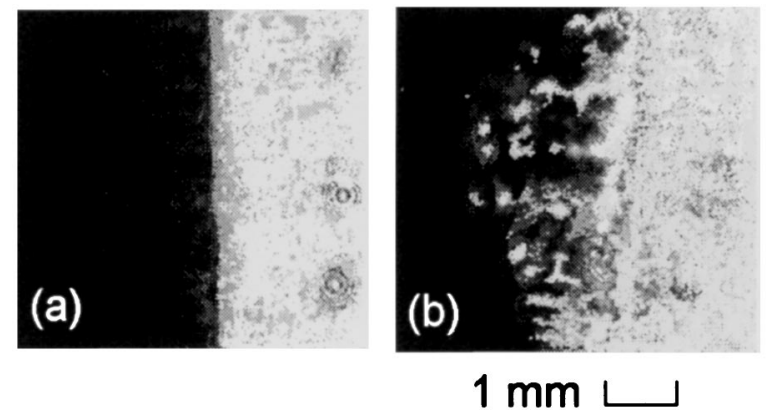

FIG. 2. Two images of rear face of crystal viewed in diffracted He-Ne laser beam: (a) a sharp boundary between steady grating and shadow areas is clearly seen; (b) bright protuberances indicate the penetration of SCW into shadow area under resonance conditions. out of resonance [Fig. 4(a)]. It is clearly seen that under resonance conditions the diffracted light completely fills the shadow area of the crystal [Fig. 4(b)]. An estimate based on the depth of SCWs penetration gives a value for the quality factor of $Q>100$.

Figure 3(b) shows the Ar-ion laser beams in the far field. One of the beams is a weak beam sent through the crystal in the direction of maximum amplification. The quality factor $Q$ was determined with higher accuracy by measuring the decay time $\tau_{g}$ of the grating after the weak beam was cut off in front of the crystal. Outside resonance $\tau_{g}$ was found to be $15 \mathrm{~ms}$, which coincides with the Maxwell relaxation time for an incident intensity of $10 \mathrm{~mW} / \mathrm{cm}^{2}$. In resonance $\tau_{g}$ was found to be $1.2 \mathrm{~s}$, which is about seven times higher than in the case of the planar configuration. At a measured velocity $v_{0}=$ $420 \mu \mathrm{m} / \mathrm{s}$ and a grating spacing of $23 \mu \mathrm{m}$ we obtain $Q \approx 140$ for the nonplanar and $Q \approx 20$ for the planar geometry.

A larger quality factor for SCWs running in a certain direction indicates a higher amplitude of the corresponding running refractive-index grating, which is shifted $\pi / 2$ with respect to the interference field, and consequently to a higher amplification of a weak beam. Under some circumstances the threshold for self-oscillation may be exceeded, resulting in the appearance of a spontaneous beam in the direction of maximum amplification. This explains other experiments where strong scattering in bismuth silicate was observed in a particular direction $[15,16]$.

To analyze self-oscillation with only one incident beam, suppose we do have a light interference pattern with a small modulation $m \ll 1$ at the input face of the crystal. The origin of a weak light wave with an amplitude $A_{1}$ interfering with the strong incident wave $A_{0}\left(A_{0}^{2}=\right.$ $I_{0}$ ) will be explained below; the only important thing now is that a frequency shift exists between the weak and the strong wave resulting in the continuous motion of the interference fringes with the resonance velocity $v_{0}$. The amplitude of the SCW is $E_{\mathrm{sc}} \approx m E_{0} Q / 2$, and

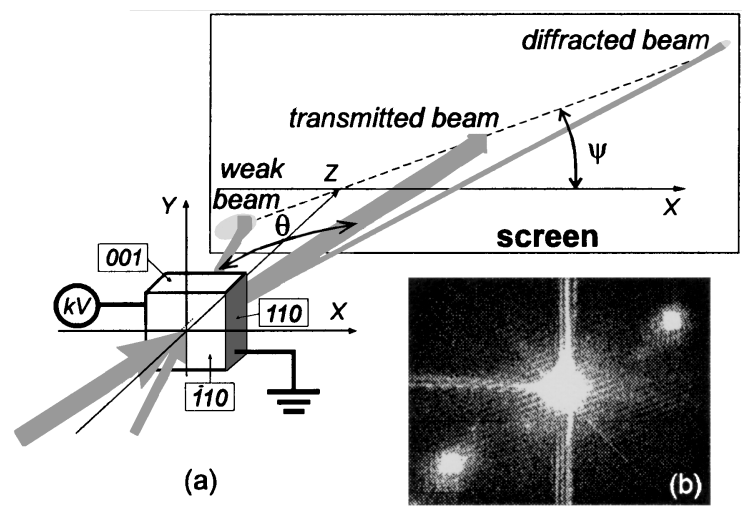

FIG. 3. (a): Nonplanar configuration where plane of incidence of two beams is tilted with respect to applied field $E_{0} \|\langle 110\rangle$ crystal axis at an angle $\psi$. (b) Far field view of Ar laser beams. 

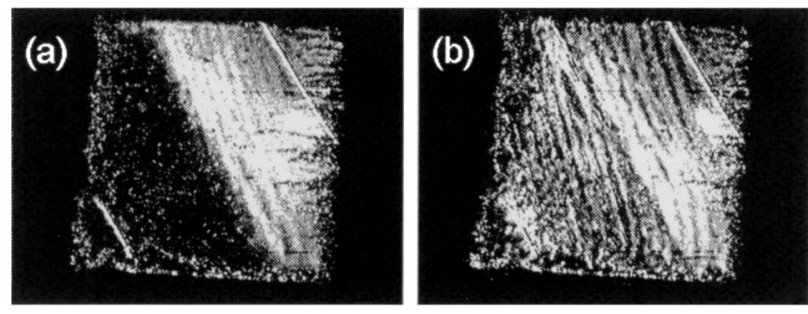

FIG. 4. The penetration of SCW into a shadow area for nonplanar configuration. Both images viewed in diffracted HeNe laser beam: (a) out of resonance; (b) in resonance. All the shadow area is illuminated at resonance.

the refractive-index grating amplitude is $G=r_{\mathrm{eff}} n^{3} E_{\mathrm{sc}}$, where $r_{\text {eff }}$ is the effective electro-optic coefficient and $n$ is the refractive index. The weak beam is amplified due to coupling with the strong beam and is attenuated due to absorption described by the coefficient $\alpha$ :

$$
\begin{aligned}
\frac{d A_{1}}{d z} & =\frac{2 \pi}{\lambda} G(z) A_{0}-\alpha z \\
& =\frac{2 \pi r_{\mathrm{eff}} n^{3}}{\lambda} \frac{E_{0} Q}{2} \frac{A_{0}^{2} A_{1}}{A_{0}^{2}+A_{1}^{2}}-\alpha z,
\end{aligned}
$$

which gives exponential growth of the weak wave amplitude $\left(A_{1} \ll A_{0}\right)$ in the case of amplification prevailing on absorption: $A_{1}(z)=A_{1}(0) \exp (\Gamma z-\alpha z)$, where $\Gamma=\pi r_{\text {eff }} n^{3} Q E_{0} / \lambda$ is the amplification factor. Note that due to the Doppler effect the frequency of wave $A_{1}(z)$ is shifted with the value $\delta \omega=-v_{0} K_{0}$.

For self-starting oscillation a positive feedback is necessary. Such a feedback may be caused by reflections of the amplified weak beam at an output and input faces of a crystal. After two reflections with proper phase conditions we have an amplitude

$$
A_{1}(0)=R_{1} R_{2} A_{1}(0) \exp (\Gamma d-2 \alpha d) .
$$

where $R_{1}$ and $R_{2}$ are amplitude reflection coefficients at the input and output faces, and $d$ is the crystal thickness. Assuming $R_{1} \cong R_{2} \cong R$, the threshold condition is determined from (3) as $R^{2} \exp \left(\Gamma_{\mathrm{th}} d-2 \alpha d\right)=1$ or $\Gamma_{\text {th }}=2(\alpha d-\ln R) / d$. For a $4 \mathrm{~mm}$ thick crystal with antireflection coatings on the faces $(R=0.2)$ and absorption $\alpha=2.8 \mathrm{~cm}^{-1}$, we find $\Gamma_{\mathrm{th}} \approx 14 \mathrm{~cm}^{-1}$, and the corresponding value of $Q_{\text {th }}$ amounts to $5.6(n=2.615$ and $\left.r_{\text {eff }}=3.7 \mathrm{pm} / \mathrm{V}\right)$. This rather low value of $Q_{\text {th }}$ indicates that it should be easy to reach self-oscillation. However, additional losses (scattering from impurities, reduced coupling due to optical activity, saturation of gain, and nonparallel faces of the crystal) result in a much higher value of $Q_{\text {th }}$.

To reach self-oscillations, we took crystal without antireflection coatings on the faces with a resulting higher value of the amplitude reflection coefficient $R(40 \%$ instead of 20\%) and with smaller transverse dimension $(6 \mathrm{~mm})$, which with the same applied voltage results in higher electric field $E_{0}$. With this crystal we found sharp threshold for self-oscillation with an applied electric field of $5.3 \mathrm{kV} / \mathrm{cm}$.

Left circular polarization of incident beam produces two strong scattered beams as shown in Fig. 5(a). (The pictures was taken at a distance of $4 \mathrm{~m}$ behind the crystal.) The angle of incidence is $14 \mathrm{mrad}$ and the angle $\psi$ measured between the electric field direction and the plane of incidence is $130^{\circ}$. Right circular polarization of the incident beam produces a picture as shown in Fig. 5(b). The angle of incidence is now $14 \mathrm{mrad}$ and $\psi=40^{\circ}$. In general (arbitrary elliptical or linear state of polarization) four wide speckle-like scattering spots locate symmetrically around the transmitted beam. The wavelength of the self-excited SCW calculated from the angular separation between the transmitted and the spontaneous beam was found to be $26 \mu \mathrm{m}$ and the divergence of the self-oscillating beam was $10 \mathrm{mrad}$ along the $y$ axis and $4 \mathrm{mrad}$ along the $x$ axis, respectively. The power of the self-oscillating beam was measured to about $10 \%$ of the transmitted beam. The self-oscillating beam was found to have a frequency shift relative to the primary one of $16 \mathrm{~Hz}$. This is close to the resonance frequency for SCWs excitation (for $10 \mathrm{~mW} / \mathrm{cm}^{2}$ we obtain a resonance frequency of $16.4 \mathrm{~Hz}$ ).

Within each lobe of the scattering pattern we observe a distinct dynamic spatial structure with movement in the direction against that of the motion of interference fringes (Fig. 6) when viewing the self-oscillating beam in the near field with $25 \times$ magnification. This is an indication of a SCWs group velocity in the opposite direction of the phase velocity of the refractive index
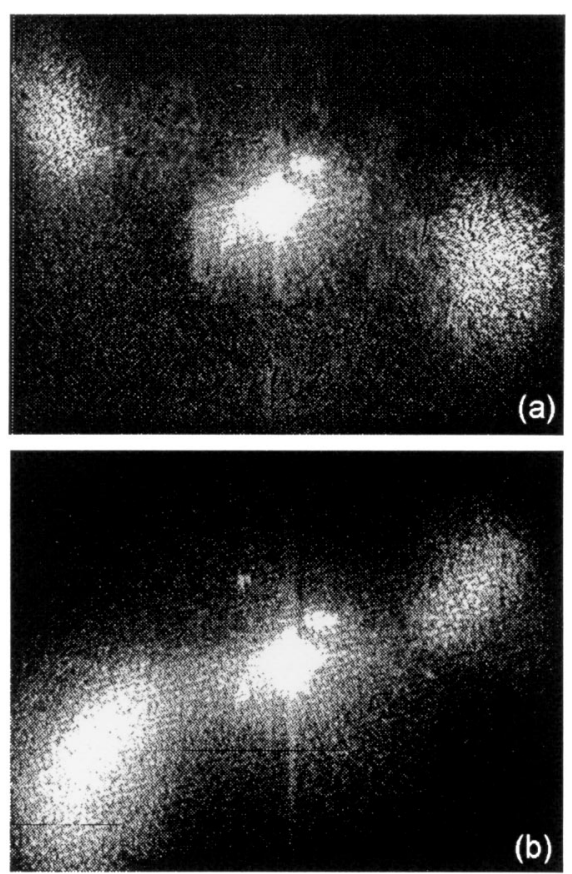

FIG. 5. Self-oscillating beams excited by circularly polarized incident beam: (a) left circular polarization; (b) right circular polarization. 


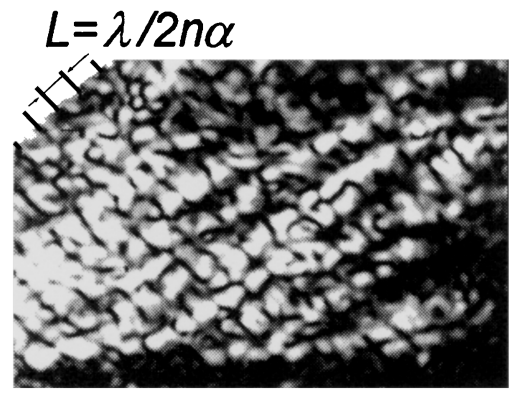

FIG. 6. Spatial structure of SCW in the crystal viewed through oscillating beam. Spatial inhomogeneities of SCW are seen as a set of crests on the background of dark stripes caused by wedge $\alpha \approx 1 \mathrm{mrad}$ of the sample (measured spacing $L \approx 100 \mu \mathrm{m}$ coincides with the calculated $L=\lambda / 2 n \alpha)$.

grating. This phenomenon is similar to the motion of spatial domains inside spontaneous subharmonic beams, as observed earlier [17]. This spatial inhomogeneity of the SCWs causes a speckle-like structure and a large divergence of the spontaneous beams.

In summary, we have directly detected SCWs in a photorefractive $\mathrm{Bi}_{12} \mathrm{SiO}_{20}$ crystal by their propagation from the region of the driving interference pattern into a region of uniform illumination. The experimental conditions, which cause a maximum quality factor for SCWs, have been found. Self-oscillation due to a combination of SCWs excitation and diffractive beam coupling was found to occur in the direction with maximum quality factor. In contrast to the well-known photorefractive oscillators $[18,19]$ we report here the result of self-excitation of SCWs in a particular direction which results in selfoscillation of spontaneous beams.

Sergei Lyuksyutov and Mikhail Vasnetsov are also with the Institute of Physics, National Ukrainian Academy of Science, Kiev, 252650 Ukraine. This work was supported by Danish Technical-Scientific research Council Grant No. 9400102.
*Electronic address: SERGEI@MIPS.FYS.DTU.DK

[1] For earlier works, see, e.g., A. S. Furman, Sov. Phys. Solid State 29, 1076 (1987), and references therein.

[2] Topics in Applied Physics, Photorefractive Materials and Their Applications I, edited by P. Günter and J.-P. Huignard (Springer-Verlag, Berlin, 1988), Vol. 61; N. V. Kukhtarev, Sov. Tech. Phys. Lett. 2, 438 (1976).

[3] S. I. Stepanov, V. V. Kulikov, and M.P. Petrov, Opt. Commun. 44, 19 (1982).

[4] V.A. Kalinin and L. Solymar, Appl. Phys. Lett. 68, 167 (1996)

[5] B. I. Sturman, M. Mann, J. Otten, and K. H. Ringhofer, J. Opt. Soc. Am. B 10, 1919 (1993).

[6] B. I. Sturman, E. Shamonina, M. Mann, and K.H. Ringhofer, J. Opt. Soc. Am. B 12, 1642 (1995).

[7] V.S. Liberman and B. Ya. Zel'dovich, Int. J. Nonlinear Opt. Phys. 3, 39 (1993).

[8] V.S. Liberman and B. Ya. Zel'dovich, Opt. Quantum Electron 25, 231 (1993).

[9] P. Refregier, L. Solymar, H. Rajbenbach, and J. P. Huignard, J. Appl. Phys. 58, 45 (1985).

[10] G. C. Valley and M. B. Klein, Opt. Eng. 22, 704 (1983).

[11] R. A. Mullen and R. W. Hellwarth, J. Appl. Phys. 58, 40 (1985).

[12] P. Buchhave, S. F. Lyuksyutov, and M. V. Vasnetsov, Opt. Lett. 20, 2363 (1995).

[13] H. C. Ellin, A. Grunnet-Jepsen, L. Solymar, and J. Takacs, in Proc. SPIE Int. Soc. Opt. Eng. 2321, 107 (1994).

[14] G. Pauliat, P. Mathey, and G. Roosen, J. Opt. Soc. Am B 8, 1942 (1991).

[15] H. Rajbenbach, A. Delboulbe, and J. P. Hiugnard, Opt. Lett. 14, 1275 (1989).

[16] H. C. Pedersen, P.E. Andersen, and P. M. Johansen, Opt. Lett. 20, 2475 (1995).

[17] P. Buchhave, S.F. Lyuksyutov, M. V. Vasnetsov, and C. Heyde, J. Opt. Soc. Am. B 13, 2595 (1996).

[18] H. Rajbenbach and J.P. Huignard, Opt. Lett. 10, 137 (1985).

[19] S. Odoulov, M. Soskin, and A. Khizhnyak, Optical Oscillators with Degenerate Four-Wave Mixing (Harwood Academic Publishers, London, 1989). 